\section{IBD's bacteria cafeteria}

\section{By Kai-Jye Lou, Staff Writer}

The challenges of delivering the protease inhibitor elafin to the gut have stymied efforts to use this anti-inflammatory protein as a therapeutic for intestinal inflammation. Now, researchers in France have engineered food-grade lactic acid bacteria that secrete elafin. ${ }^{1}$ Oral delivery of the engineered bacteria reduced inflammation and restored intestinal homeostasis in mouse models of inflammatory bowel disease.

ViThera Pharmaceuticals Inc. holds a license to the technology and thinks it will be ready to move the engineered bacteria into a Phase I trial by late 2014 in patients with mild to moderate ulcerative colitis (UC).

Elafin is a protease inhibitor that targets elastase and proteinase-3. In the gut of healthy individuals, elafin is primarily produced by intestinal epithelial cells. Downregulated elafin expression and elevated proteolytic activity in the gut are linked to Crohn's disease and UC, the two major forms of IBD. ${ }^{2-4}$

In 2011, a French research group led by Nathalie Vergnolle published data from genetic studies in mice suggesting elafin can protect against intestinal inflammation. ${ }^{5}$

"Elafin is a multifunctional protein with multiple protective properties that make it a good choice for use in IBD," said Vergnolle, deputy director of The Physiopathology Center of Toulouse-Purpan at the Institut National de la Santé et de la Recherche Médicale (INSERM). "The protein can restore the balance of intestinal proteolytic activity, inhibit the recruitment of inflammatory cells and their release of proinflammatory factors, has antimicrobial properties and also could help re-equilibrate the gut microflora."

The problem is that digestive processes in the gut destroy elafin, and the layer of mucus lining the gut acts as a physical barrier that prevents the protein from efficiently reaching its site of action at the gut epithelium. Delivering high concentrations of the protein to the gut would be impractical in IBD and also could interfere with digestion.

The group thus looked to the intestinal microflora to supplement the gut mucosa with a steady supply of elafin. To do so, the team settled on two strains of lactic acid bacteria-Lactococcus lactis and Lactobacillus casei-as vectors for elafin delivery. These bacteria are adapted to survive in the gut microenvironment and have a generally recognized as safe (GRAS) status with the FDA.
"Lactic acid bacteria are known to colonize the gut when ingested and are found in the normal intestinal microflora," Vergnolle told SciBX. "Thus we sought to transform the bacteria to have them produce and secrete elafin."

In a mouse model of chemically induced colitis, oral delivery of elafin-secreting L. lactis or L. casei restored proteolytic homeostasis and reduced gut inflammation, whereas wild-type strains did not. In human colon epithelial cell culture, elafin-secreting L. lactis protected the cells from inflammation-induced loss of barrier function, whereas wild-type L. lactis did not. L. casei was not evaluated in the latter study.

Results were published in Science Translational Medicine. Philippe Langella, a research director at the French National Institute for Agricultural Research (INRA) and cofounder of ViThera, was a coauthor of the study.

"Our data show that we can very significantly reduce inflammation in the gut by treating mice orally with our engineered lactic acid bacteria," said Vergnolle, the corresponding author.

\section{Benefits of bacteria}

ViThera founder and president Johannes Fruehauf said that the data demonstrate the utility of the company's EnLact platform, which uses engineered lactic acid bacteria to deliver therapeutic proteins to the gut.

"In our mind, engineered lactic acid bacteria are the only way to get effective delivery of therapeutic proteins to the intestinal epithelium," he told SciBX. "We don't think capsule-based delivery of elafin itself, or any other therapeutic protein, would be very effective because the protein will be released into the intestinal lumen and will still need to get through a mucous barrier before it reaches the intestinal epithelium. The intestine is rich in proteases and because of this, very little of the protein would be able to reach the intestinal epithelium before it is destroyed."

Fruehauf noted that the engineered bacteria described in the paper attach to the intestinal epithelium, which is the site where elafin needs to be delivered. He also said secreted elafin acts locally and is unlikely to have systemic effects.

Fruehauf added that systemically delivered antiprotease therapies are known to be associated with significant side effects such as joint toxicity.

He thinks the engineered bacteria could be used in many types of IBD. "I think our approach can be applied across the entire range of IBD patients, from those who have very severe disease to those who have very mild disease, and possibly even in those whose disease is in remission," he said. "Current data in IBD patients show that there is still unbalanced proteolytic activity in the noninflamed regions of the gut, so we may be able to use our engineered bacteria to restore that balance."

The company uses fermentation to produce large quantities of the engineered bacteria. The purification step "is a simple and cost-effective process involving filtration," according to Fruehauf. 


\section{ANALYSIS}

\section{Restore and reduce}

Fruehauf and Vergnolle both think elafin-secreting bacteria could be used together with marketed IBD drugs such as glucocorticoids, methotrexate and anti-tumor necrosis factors (TNFs) as an adjunctive therapy to restore intestinal functionality.

Existing drugs that reduce inflammation by suppressing the immune system also increase the risk of serious infections and cancer. Moreover, glucocorticoids can disrupt lipid and glucose metabolism.

"For now, we think our approach using these engineered bacteria could supplement existing therapies to treat IBD but won't necessarily replace them," said Fruehauf. "If our approach works, it could be used in conjunction with current treatments to help push the disease into remission, prolong the time the disease stays in remission and reduce the need for immunosuppressive drugs in patients."

ViThera is developing an attenuated, nondisseminating strain of elafin-secreting $L$. casei for use in humans. The product likely will involve freeze-dried bacteria in a capsule with a polysaccharide coating that facilitates targeted release in the large intestine. He said the company opted for engineered L. casei over L. lactis because the former is better at attaching to the intestinal epithelium.

INSERM, INRA, the Pasteur Institute and Paris Diderot University have cofiled for a patent covering recombinant probiotic bacteria to treat and prevent IBD and irritable bowel syndrome (IBS). The IP is licensed to ViThera.

Lou, K.-J. SciBX 5(44); doi:10.1038/scibx.2012.1154

Published online Nov. 8, 2012

\section{REFERENCES}

1. Motta, J.-P. et al. Sci. Transl. Med.; published online Oct. 31, 2012; doi:10.1126/scitransImed.3004212

Contact: Nathalie Vergnolle, Institut National de la Santé et de la Recherche Médicale (INSERM), Toulouse, France e-mail: nathalie.vergnolle@inserm.fr

2. Schmid, M. et al. J. Leukoc. Biol. 81, 907-915 (2007)

3. Cenac, N. et al. J. Clin. Invest. 117, 636-647 (2007)

4. Eriksson, A. et al. BMC Gastroenterol. 8, 34 (2008)

5. Motta, J.-P. et al. Gastroenterol. 140, 1272-1282 (2011)

\section{COMPANIES AND INSTITUTIONS MENTIONED} Institut National de la Santé et de la Recherche Médicale Toulouse, France French National Institute for Agricultural Research, Paris, France

Paris Diderot University, Paris, France

Pasteur Institute, Paris, France

ViThera Pharmaceuticals Inc., Cambridge, Mass. 